\title{
RSOBIA - A new OBIA Toolbar and Toolbox in ArcMap 10.x for Segmentation and Classification.
}

\author{
T.P. Le Bas
}

National Oceanography Centre, European Way, Southampton, UK. SO14 3ZH.

tim.lebas@noc.ac.uk

KEY WORDS: OBIA, Segmentation, ArcMap, Classification, Toolbar

\begin{abstract}
:
A new toolbox for ArcMap 10.x will be presented that segments the data layers into a set of polygons. The software is available at htttp://www.codemap.eu/Outputs . Each polygon is defined by a K-means clustering and region growing algorithm, thus finding areas, their edges and boundaries in the imagery. Attached to each polygon are the characteristics of the imagery such as mean and standard deviation of the pixel values, within the polygon. The segmentation of imagery into a jigsaw of polygons also has the advantage that the human interpreter does not need to spend hours digitising the boundaries. The actual segmentation process has been taken from the RSGIS library of analysis and classification routines (Bunting et al., 2014). These routines are freeware but have been modified to be made available in the ESRI ArcGIS software under the Windows operating system. Input to the segmentation process is a multi-layered raster image, for example; satellite imagery, or any set of raster datasets made up from derivatives of topography. The size and number of clusters are set by the user and are dependent on the imagery used.
\end{abstract}

The advantage of having OBIA within the ArcGIS environment is that it can become part of the workflow, either separately or in models. Such integration speeds analysis and allows easier manipulation of data. Meaningful classification of the polygons using their numerical characteristics can be very dependent on the data subject. Many classification systems are available and tailored to the data available. A simple classification tool is provided as a paint function, but it is expected that functionality within ArcMAP is already being used to undertake complex classification rules.

\section{INTRODUCTION}

OBIA (Object Based Image Analysis) is a relatively new technique that mimics human interpretation of imagery. The objectivity and speed of production of results aids the interpreter to choose either the most favourable results from a subjective point of view or the use of statistical parameters and measures for a quantified interpretation.

Software for OBIA is available (e.g. eCognition made by Trimble). The issue with much of these software is the learning curve required to produce useful and recognisable results. If users are willing and able to spend the time learning how to use such software the results can be excellent. An additional issue however is that the data has to be exported from their source software and imported into the OBIA software, followed by any results exported from the OBIA software back into the source software. Often this source software is a GIS system such as ESRI's ArcMap.

A need was therefore seen for OBIA to be integrated into GIS. ESRI's ArcMap is currently used by millions of people daily worldwide and could benefit from access to OBIA. Easy usage and access is essential. A toolbar for push button control was envisaged and RSOBIA (Remote sensing Object Based Image
Analysis) was created. The toolbar is easy to use and requires very little training. For the slightly higher level user a toolbox has also been created, so that the RSOBIA functions can be incorporated into other programming models and allows more complex functions to be created.

\section{SOFTWARE}

The RSOBIA toolbar is added to the ESRI ArcMap version 10.x toolbars. It consists of 3 sections. The first section is designed for use with single band imagery and creates mathematical derivatives of the single band imagery, such as slope maps from topography. The second section has the main segmentation algorithms creating polygonised feature data. The input for segmentation is either multi-band imagery, such as Landsat imagery and RGB pictures, or a combination of single layer grids making a multi-layered dataset. The final section in RSOBIA is for classification and interpretation of the polygonised features.

\subsection{Derivatives}

There are 5 derivatives currently available. The functions offered by the derivatives menu are essentially standard grid manipulation techniques but brought together for ease of use. 


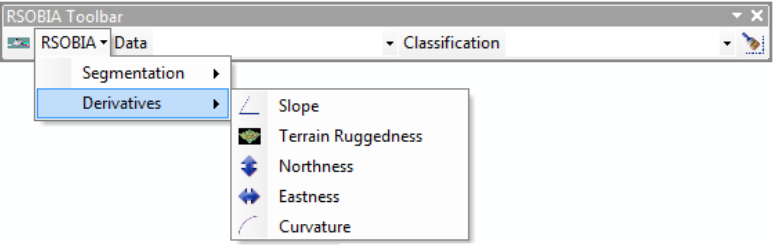

Figure 1: RSOBIA toolbar showing the derivatives sub-menu

\subsubsection{Slope}

This calculates the maximum slope (in any direction) in degrees. Values created are real numbers between 0.0 and 90.0 but a value of -1.0 is used for areas of no dataThis function differs from a shaded relief which is a slope derivative from a particular direction. Slope maps are occasionally used for pseudo-3D display - using the greyscale slope map with the high slope as black, and placed under a colour topography map with partial transparency.

\subsubsection{Terrain Ruggedness}

This is sometimes referred to as Rugosity. Calculates the variation in bathymetry datasets within a neighbourhood. This technique combines the variability of slope and aspect in an area. It is taken from the "Benthic Terrain Modeller" developed by Shaun Wallbridge (Wright et al., 2012).

\subsubsection{Northness}

This is the calculation of the aspect of the slope. Aspect is often calculated is terms of the compass direction 0-360 degrees. For terrain calculations the zero and 360 degree boundary can create issues and so the Northness is calculated being the cosine of the aspect. Values are from 1.0 (fully north facing) to -1 (south facing).

\subsubsection{Eastness}

This is the complement to Northness being the sine of the Aspect. Values are from 1.0 (fully east facing) to -1 (west facing).

\subsubsection{Curvature}

The calculation of the curvature is in the direction of maximum slope of a bathymetry dataset. It is taken from the spatial analyst toolbox. This is useful for highlighting crests and depressions. Values often are between 30.0 (convex crest) and -30.0 (concave depressions).

\subsection{Segmentation}

The segmentation section of the toolbar covers more than just segmentation. There are 5 tools available: layering of single band grids together, segmentation to create polygonised maps, polygon attribute calculations, seeding of polygons with groundtruth point sample attributes and a bespoke wizard tool for multibeam bathymetry and backscatter interpretation.

\subsubsection{Layering}

The layering function is a preparation step for segmentation. This function can combine many single layered files as required and create a multi-layered raster image.

\subsubsection{Segmentation}

Segmentation is the method to aggregate pixels together to create a thematic map. The segmentation process is a licensed tool taking a multi-layered image and creates a set of polygons defined by the statistics associated with the layered image. Clusters of the imagery pixels are created in n-dimensional space and created into classes. Aggregation into geographic regions (polygons) is done according to a minimum polygon size rule, and clustering rules.

There are 3 main parameters required:

i. Number of Clusters: This is the most important parameter and depends on the complexity of the data.

ii. Minimum Object Size: This is the minimum size of any output polygon in terms of pixels. Knowledge of the pixel resolution is crucial.

iii. Layer Weights: During the standard segmentation process each layer is given equal weighting regardless of the differing units used on each layer. The user can provide individual layer weights if, for example, one layer is deemed to provide more important or better imagery.

The segmentation process uses a technique of k-means clustering and uses the iterative removal of outliers (Shepherd et al., 2014). This technique is incorporated in a large software library RSGISLib (Bunting et al., 2014). The library is accessed by the RSOBIA toolbar.
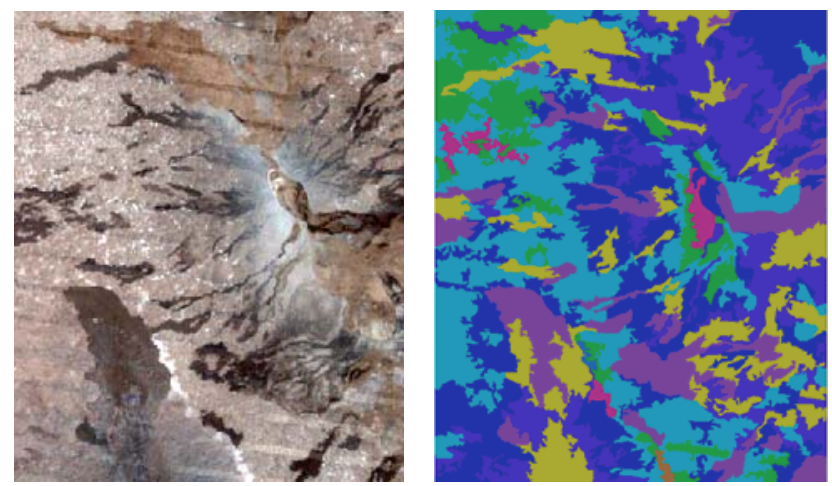

Figure 2. Left: Layered Landsat Imagery (4 layers, Bands 4,3,2 in RGB), Right: Results of segmentation using 10 classes.

\subsubsection{Adding of Attributes}

Following segmentation, the attributes for each polygon only relate to the polygon shape and k-means class. It is often required to view and use the values of initial raster layers. These can be aggregated for each polygon as an attribute value of the mean and standard deviation of the pixel values within the polygon for each layer. 


\subsection{4. $\quad$ Adding of Groundtruth Samples}

The attributes for each polygon can be extended if groundtruth point data is available. This is a very simple join of two datasets and effectively adds the attributes of a groundtruth data point to the relevant polygon. In this way the samples may be utilised to characterise the class type.

\subsubsection{Multibeam Echosounder (MBES) Wizard}

A specific wizard is available for multibeam echosounder bathymetry and backscatter imagery. It is designed to carry out a series of operations that are all available in the RSOBIA toolbar. The advantage of this is an easier and quicker method for the novice user. The wizard tool has been designed to have very few input parameters: the bathymetry and backscatter grids, the number of classes, the minimum size of the polygon and an output file. The wizard is programmed to work at any pixel resolution and will produce results at the resolution of the largest pixel of the two input files.

\subsection{Classification}

A manual classification tool is available for labelling polygons with a class name (e.g. for marine habitat mapping "Sandy Mud”). The user can choose pre-defined classification class names or type in their own class name.

The user can "paint" polygons with the appropriate colour and each polygon selected will be attributed the classification name (for example Sandy Mud).

\section{INSTALLATION}

The RSOBIA toolbar is an "addin” for ESRI's ArcMap 10.x. The Spatial Analyst extension is also required. Installation is generally a two stage setup. Two files are required: RSOBIA_setup.exe (about 138Mb) and RSOBIA.esriaddin (about 350Kb). These are both available from http://www.codemap.eu/Outputs together with full documentation.

Installation is designed to be quick and easy for the user. RSOBIA requires Python and specialised Windows libraries to be installed together in the same place. When installation is complete you should get the splash screen confirming success (Figure 5)

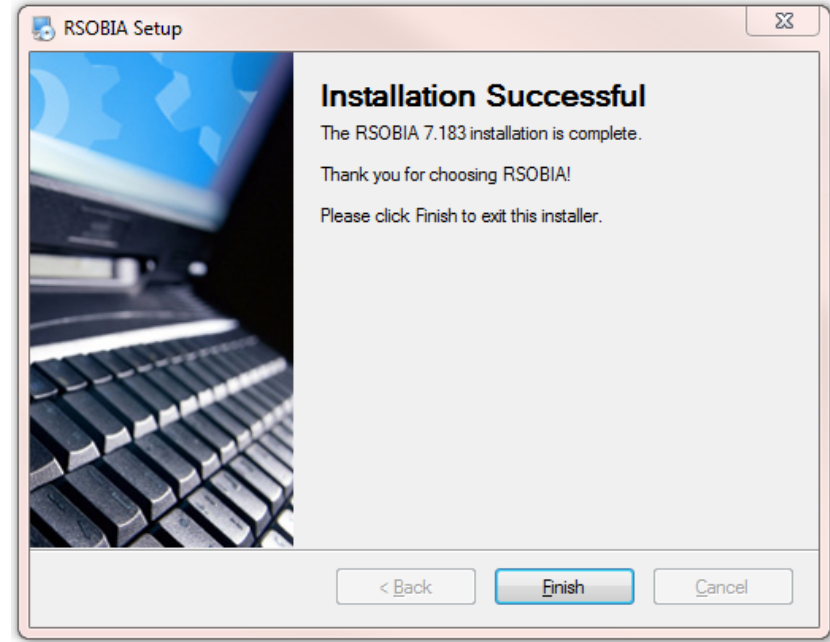

Figure 3: Splash screen after successful installation of the RSOBIA library functions

\section{USAGE}

The toolbar can be used on all types of data. Thematic maps from imagery data are often desired and the speed and reliability of objective mapping makes the RSOBIA toolbar a useful addition to ArcMap. Its ease of use is key. Testing has been done on Landsat imagery, Lidar data, Photogrammetric imagery and photographs and digital colour scans. Figure $\mathrm{X}$ shows versatility of the tool providing the segmentation from a very famous painting.
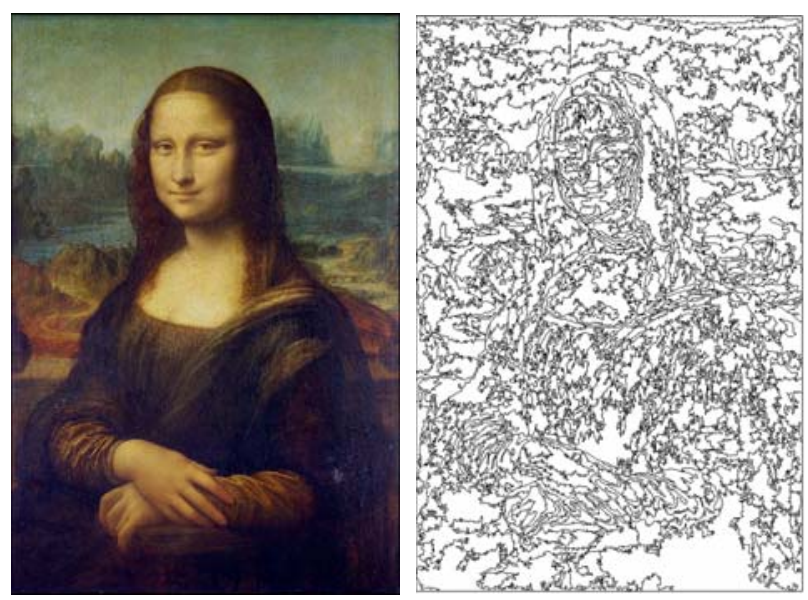

Figure 4: Example of the versatility of the segmentation process. Original imagery was scanned in 3 colours (1024 by 687 pixels) and the results of segmentation using 30 clusters (colours).

It has also very successfully been used on compilation datasets. Data from different data streams and sources have been combined by layering the data into a layered image. This layered image has then been segmented and has created a thematic map. The classes found were then classified using ground truth data and a final map produced.

If the segmentation results are not pleasing to the eye or seem to omit features, the parameters can be set differently and the process can be repeated. 


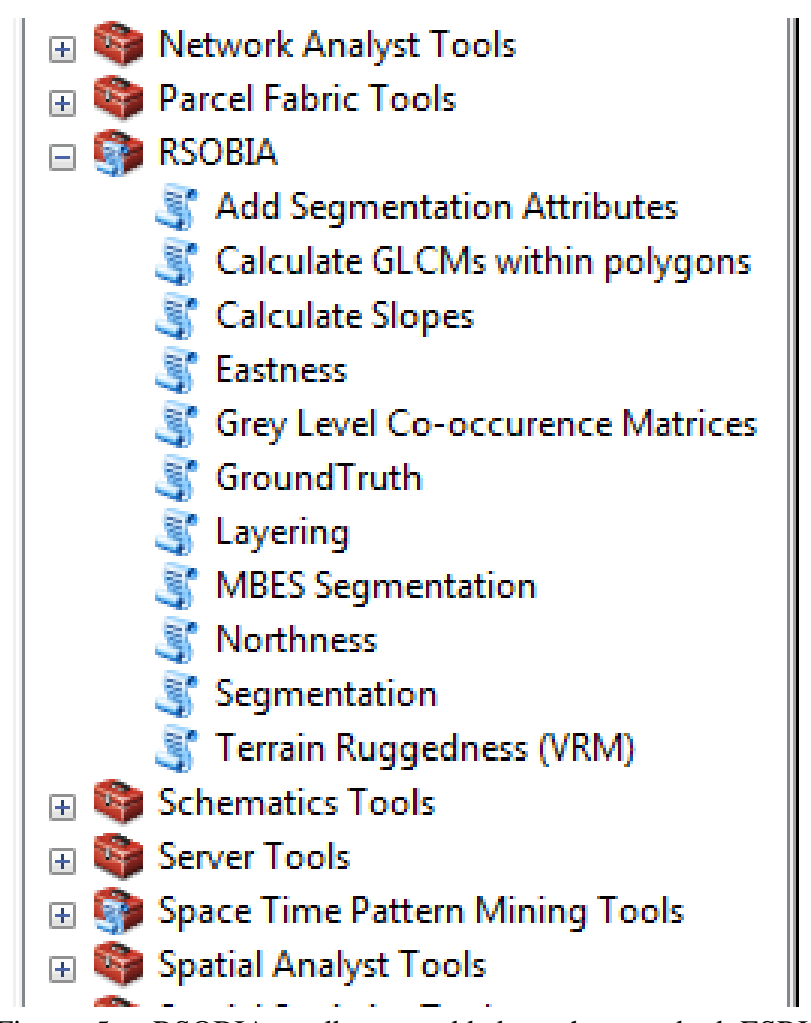

Figure 5: RSOBIA toolbox - added to the standard ESRI toolboxes.

A second way of using the tools is via the toolbox (see Figure 5 and 6). Users can create their own models using the RSOBIA tools together with standard ESRI tools. The model builder is very versatile and in this way the RSOBIA tools can be incorporated into user defined processes and data protocols.

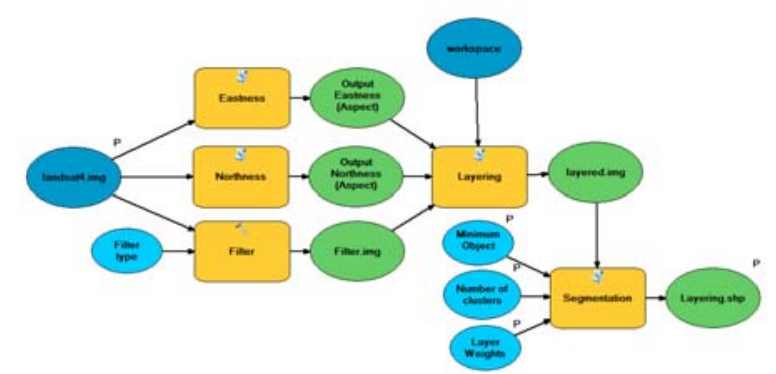

Figure 6: Example of the usage of the RSOBIA tools in a bespoke model using the model builder in ArcMap.

\section{CONCLUSIONS}

The RSOBIA toolbar is a new addition to the ArcMap set of tools. Object Based Image Analysis is a useful technique for analysis and interpretation of raster data and can be used on imagery and gridded data. Manual interpretation is slow, resource intensive and subjective. OBIA results are objective, repeatable and fast. The advantage of RSOBIA is that the process can be integrated into current workflows, and this improves efficiency of multiple processing results. The tools have been designed to be easy to learn and implement.

\section{REFERENCES}

Bunting, P.; Clewley, D.; Lucas, R.M.; Gillingham, S. The Remote Sensing and GIS Software Library (RSGISLib). Comput. Geosci. 2014, 62, 216-226.

Calinski, T., and J. Harabasz. "A dendrite method for cluster analysis." Communications in Statistics. Vol. 3, No. 1, 1974, pp. $1-27$.

Haralick, R.M., K. Shanmugam and I. Dinstein. Textural Features for Image Classification. IEEE Transactions on Systems, Man and Cybernetics. 1973 SMC vol. 3, no. 6, pp.610620.

Shepherd, J.D.; Bunting, P.; Dymond, J.R., Operational largescale segmentation of imagery based on iterative elimination. J. Appl. Remote Sens. 2014, in press.

Wright, D.J., Pendleton, M., Boulware, J., Walbridge, S., Gerlt, B., Eslinger, D., Sampson, D., and Huntley, E. 2012. ArcGIS Benthic Terrain Modeler (BTM), v. 3.0, Environmental Systems Research Institute, NOAA Coastal Services Center, Massachusetts Office of Coastal Zone Management. Available online at http://esriurl.com/5754. 